\section{Comitês de prevenção da mortalidade infantil no Paraná, Brasil: implantação e operacionalização}

\author{
Committees for the prevention of infant \\ mortality in the State of Paraná, Brazil: \\ implementation and operation
}

Nereu Henrique Mansano ${ }^{1}$ Verônica de Azevedo Mazza 1,2 Vânia Muniz Nequer Soares 1 Maria Aparecida Rapozo Araldi 1,3 Vera Lúcia Moreira Cabral 1,3

\title{
Introdução
}

1 Comitê Estadual de Prevenção da Mortalidade Infantil, Secretaria de Estado da Saúde do Paraná, Curitiba, Brasil.

2 Departamento de Enfermagem, Universidade Federal do Paraná,

Curitiba, Brasil.

3 Departamento

de Enfermagem, Pontifícia Universidade Católica do Paraná, Curitiba, Brasil.

Correspondência Verônica de Azevedo Mazza Comitê Estadual de Prevenção da Mortalidade Infantil, Secretaria de Estado da Saúde do Paraná. Rua Piquiri 170 , Curitiba, $P R$ 80230-140, Brasil. vcmazza@ig.com.br

\section{Abstract}

This article reports on the implementation and operation of committees for the prevention of infant mortality in the State of Paraná, Brazil, with the operational strategies,formation, and relations at three levels: State, regional, and municipal. To implement the committees it was necessary to train professionals to investigate infant deaths. In two years the committees analyzed $50 \%$ of the infant deaths occurring in the State. The goal is to increase the number of cases analyzed and to continue to monitor the committees' work, seeking improved performance, agility, and data quality.

Infant Mortality; Epidemiologic Surveillance; Professional Staff Committees
A mortalidade infantil tem sido freqüentemente apontada como indicador sensível da qualidade de vida de uma população 1,2, determinada em sua dimensão mais ampla pelas condições sociais, econômicas e culturais dos indivíduos e da comunidade a que pertencem 1 .

No Paraná, a mortalidade infantil tem apresentado tendências declinantes nos últimos anos. Segundo o Sistema de Informação de Mortalidade (SIM), em 1994 essa taxa foi de 25,89/ mil nascidos vivos (NV) e, em 2001, de 17,40/ mil NV, apresentando uma redução de 48,8\%.

Em 1999 foram implantados no Estado os Comitês de Prevenção da Mortalidade Infantil, com o intuito de reduzir ainda mais esses índices. Sarinho 3 enfatiza a importância de estudos epidemiológicos de base populacional como um instrumento para desenvolver ações no campo da saúde pública, bem como o papel da epidemiologia na avaliação da qualidade na assistência à saúde, visando à incorporação do enfoque de risco tanto na dimensão individual como na coletiva.

Os comitês têm a finalidade de investigar cada óbito, procurando determinar a evitabilidade e, nessa hipótese, especificar medidas de prevenção. Óbitos evitáveis são aqueles que, considerando-se a ciência e a tecnologia existentes atualmente, poderiam ter sido poupados ou não deveriam ter ocorrido, segundo con- 
ceito da Associação Médica Americana adotado pelos Comitês de Prevenção da Mortalidade Materna e Infantil do Paraná (CEPMI). Óbitos no primeiro ano de vida têm sido considerados como eventos-sentinela na avaliação da qualidade da assistência e como eventos desnecessários ou consentidos 4 . A ocorrência do óbito parte da existência de uma falha no produto da atenção em saúde, exigindo uma investigação que identifique os fatos que contribuíram para esse resultado negativo (Rutstein, 1976, apud Leite et al. 5. Portanto, o trabalho dos comitês pode ter impacto direto nas mortes evitáveis.

A análise do óbito infantil e da assistência prestada serve como indicador do nível de qualidade e de acesso aos serviços de saúde, além de possibilitar conhecimento e intervenção nessa realidade de forma rápida 6 . Essa estratégia baseia-se na busca da eqüidade, podendo identificar grupos populacionais de maior risco aos quais deve ser dirigida uma atenção diferenciada e interferindo positivamente no processo de produção da saúde, da doença e da mortel. A análise evidencia, ainda, como e por que morrem os menores de um ano, e identifica os grupos que apresentam maior risco de adoecer em determinadas regiões, contribuindo, assim, para o alcance dos índices recomendados pela Organização Mundial de Saúde.

Compreender melhor o papel das atividades de assistência à saúde no processo de determinação do óbito infantil é uma necessidade e um compromisso ético 7 . Nessa perspectiva, este trabalho tem por objetivo relatar a experiência de implantação e funcionamento dos CEPMI no Estado do Paraná.

\section{Histórico dos comitês no Paraná}

A implantação dos CEPMI teve início em 1994, com a nomeação de membros e elaboração do regimento interno. Sua formalização, no entanto, só ocorreria em 1999, por meio de Resolução Estadual, após sofrer uma reorganização em 1997.

Em 1998, foi realizado o I Treinamento de Multiplicadores para Investigação dos Óbitos Infantis e Organização dos Comitês Regionais. A capacitação dos membros dos comitês regionais e municipais foi feita de acordo com sua participação no processo: treinamento para análise de causas básicas, orientação à questão da abordagem das famílias na entrevista, questões éticas ao lidar com informações dessa natureza. As investigações começaram a ser realizadas pela vigilância epidemiológica dos mu- nicípios de forma mais organizada, surgindo, então, dificuldades de acesso aos prontuários hospitalares para coleta de dados. Com o intuito de superar tais obstáculos, foi criada uma resolução determinando que hospitais e maternidades disponibilizassem cópias dos prontuários aos serviços de epidemiologia das secretarias municipais.

Em 2000, foi realizado o I Encontro dos Comitês de Prevenção da Mortalidade Infantil do Paraná, no qual foram apresentadas as investigações realizadas em 1999 e identificadas as dificuldades no primeiro ano de atuação dos comitês, estabelecendo novas estratégias para sua operacionalização. Nesse mesmo ano foi desenvolvido o programa informatizado para digitação das análises dos óbitos infantis, denominado Sistema de Investigação da Mortalidade Infantil (SIMI), que permitiu agilizar a obtenção dos dados via "rede intranet da Secretaria Estadual de Saúde (SESA)". O programa permite várias tabulações, entre as quais número de óbitos investigados por município e regional, principais causas, evitabilidade, responsabilidade e critérios de intervenção.

\section{Funcionamento e operacionalização dos comitês}

O CEPMI do Paraná é consultivo e de assessoria técnica, tendo como objetivos: orientar os comitês regionais e municipais; consolidar os dados, promovendo a avaliação contínua das mudanças nos índices de mortalidade infantil e dos fatores que as provocam; elaborar e divulgar boletins informativos e relatório analítico; e apresentar propostas de medidas de intervenção, estimulando as autoridades competentes a instituir medidas de prevenção e controle.

Tem caráter ético, confidencial e interinstitucional, contando com representação das universidades, da Secretaria de Saúde, dos conselhos de classe, de ONGs, de sociedades científicas e com o apoio técnico-administrativo do Centro de Informações e Diagnóstico em Saúde (CIDS) da Secretaria de Estado da Saúde.

Os comitês municipais procedem às investigações dos óbitos infantis utilizando como metodologia a busca ativa. Os dados são levantados por meio dos prontuários hospitalares e ambulatoriais e, quando houver, de visita domiciliar, declaração de óbito, declaração de nascido vivo, bem como de informações de agentes comunitários e de cemitérios clandestinos. Para estruturar o trabalho foram elaboradas fichas padronizadas para investigação 
Coeficientes de mortalidade neonatal, pós-neonatal e infantil, por 100 mil nascidos vivos, Paraná, Brasil, 1994 a 2001.

\begin{tabular}{lrrrrrrrr}
\hline Coeficiente de mortalidade & 1994 & 1995 & 1996 & 1997 & 1998 & $\mathbf{1 9 9 9}$ & $\mathbf{2 0 0 0}$ & $\mathbf{2 0 0 1}$ \\
\hline Neonatal & 14,64 & 13,44 & 12,44 & 11,86 & 12,55 & 12,89 & 12,79 & 11,41 \\
Pós-neonatal & 11,25 & 9,69 & 8,07 & 7,05 & 8,49 & 6,64 & 6,66 & 5,95 \\
Infantil (neonatal + pós-neonatal) & 25,89 & 23,10 & 20,40 & 18,87 & 21,01 & 19,53 & 19,44 & 17,36 \\
\hline
\end{tabular}

Fonte: Secretaria Estadual de Saúde/Instituto de Saúde do Paraná/Centro de Informações e Diagnóstico em Saúde.

dos óbitos neonatais e tardios, e uma ficha para análise final.

No momento, encontram-se em atividade 22 comitês regionais e 165 municipais. Todos os demais municípios procedem à investigação sistemática dos óbitos infantis no Paraná. Em dois anos, foram analisados 3.267 óbitos de menores de um ano, $50 \%$ do total notificado no sistema oficial de mortalidade (SIM).

\section{Situação da mortalidade infantil do Paraná}

De acordo com a Tabela 1, observa-se um declínio no coeficiente de mortalidade infantil nos últimos sete anos no Paraná, devido principalmente à redução dos óbitos pós-neonatais e, em menor parcela, dos óbitos neonatais. A queda dos óbitos pós-neonatais está comumente associada às condições de vida e saúde, aos serviços básicos de saúde e à realização de programas direcionados a situações específicas da infância, como incentivo à amamentação, imunização, acompanhamento do crescimento e desenvolvimento, além do tratamento de doenças prevalentes na infância. O componente pósneonatal é o responsável pela maior parte da redução da mortalidade infantil nas últimas décadas no Brasil, ao passo que o componente neonatal representa a maior parcela da mortalidade infantil, principalmente nas regiões onde os coeficientes são menores 8 .

\section{Considerações finais}

Os comitês realizam a investigação e a análise detalhadas dos óbitos infantis no Paraná, o que permite uma correção da causa básica no sistema oficial de mortalidade (SIM), conferindo maior credibilidade ao sistema de informação. Essa análise proporciona, ainda, a avaliação em relação à qualidade da assistência e às condições de acesso aos serviços de saúde, e expõe de maneira mais clara o panorama das condições de vida das crianças.

Durante esse período de atuação dos comitês, foi possível evidenciar as dificuldades de análise dos dados, decorrentes da falta de informações nos prontuários e da existência de registros ilegíveis pela qualidade da letra. Muitas vezes foram encontradas incongruências entre as informações dos prontuários analisados e a entrevista com familiares, suscitando o aprofundamento da análise técnica e a busca de outras fontes de informação.

Ao dar visibilidade a situações que requeriam mudanças a fim de reduzir as mortes infantis, algumas intervenções têm sido efetuadas. Entre elas, a discussão com conselhos de classe em relação à qualidade dos registros nos prontuários, no intuito de que tanto órgãos de classe quanto instituições de ensino possam interferir com ações educativas, ressaltando as regulamentações já existentes sobre o tema. São feitas, ainda, sugestões de mudanças aos gestores, com base nas deficiências do sistema de saúde apontadas nas análises.

Os CPMI do Paraná analisaram, em dois anos, $50 \%$ dos óbitos do Estado. Pretende-se ampliar gradativamente o número de óbitos analisados (60\% em 2002), bem como dar continuidade ao acompanhamento e à reestruturação dos comitês, visando a obter melhor desempenho, mais agilidade e melhor qualidade dos dados.

A experiência dos CPMI no Estado do Paraná demonstra que, para uma redução mais significativa da mortalidade infantil, é necessária uma articulação das instâncias governamentais com a sociedade civil organizada no direcionamento das novas políticas públicas de saúde para a assistência materno-infantil. 


\section{Resumo}

Este artigo tem por objetivo relatar a experiência de implantação e funcionamento dos Comitês de Prevenção da Mortalidade Infantil no Estado do Paraná, Brasil, apresentando as estratégias de operacionalização, composição e fluxo dos três níveis: estadual, regional e municipal. Para a efetiva implantação dessa proposta, foi necessário preparar profissionais para investigar o óbito infantil e, portanto, foram realizados treinamentos de Multiplicadores para Investigação dos Óbitos Infantis. Em dois anos, os comitês analisaram 50\% dos óbitos do Estado. Pretende-se ampliar gradativamente o número de óbitos analisados, bem como dar continuidade ao acompanhamento e à reestruturação dos comitês, visando a obter melhor desempenho, mais agilidade e melhor qualidade dos dados.

Mortalidade Infantil; Vigilância Epidemiológica;Comitê de Profissionais

\section{Colaboradores}

N. H. Mansano participou da etapa de coleta dos dados, organização e análise dos dados, e revisão crítica. V. A. Mazza colaborou nas etapas de organização e análise dos dados, revisão da literatura e revisão crítica. V. M. N. Soares, contribuiu na coleta dos dados, organização e análise dos dados. M. A. R. Araldi e V. L. M. Cabral, participaram da revisão de literatura e análise dos dados.

\section{Referências}

1. Aerts DRGC. Investigação dos óbitos perinatais e infantis: seu uso no planejamento de políticas públicas de saúde. J Pediatr (Rio de J) 1997; 73:364-6.

2. Szwarcwald CL, Leal MC, Castilho EA, Andrade CLT. Mortalidade infantil no Brasil: Belíndia ou Bulgária? Cad Saúde Pública 1997; 13:503-16.

3. Sarinho SW. Mortalidade pós-neonatal: novas abordagens. J Pediatr (Rio de J) 2001; 77:461-8.

4. Leite AJM. Óbitos infantis: como melhor estudálos (ou de como devemos evitá-los). J Pediatr (Rio de J) 1997; 73:362-4.

5. Leite AJM, Marcopito LF, Diniz RLP, Silva AVS, Souza LCB, Borges JC, et al. Mortes perinatais nos municípios de Fortaleza, Ceará: o quanto é possível evitar? J Pediatr (Rio de J) 1997; 73:388-94.

6. Formigli VLA, Silva LMV, Cerdeira AJP, Pinto CMF, Oliveira RSA, Caldas AC, et al. Avaliação da atenção à saúde através da investigação de óbitos infantis. Rio de Janeiro Cad Saúde Pública 1996; 12 Suppl 2:33-41.

7. Caldeira AP, França E, Goulart EMA. Mortalidade infantil pós-neonatal e qualidade da assistência médica: um estudo caso-controle. J Pediatr (Rio de J) 2001; 77:461-8.

8. Maranhão AGK, Joaquim MMC, Kalume P, Castilho O, Leal MC. Mortalidade perinatal e neonatal no Brasil. Tema 1999; 17:6-17.

Recebido em 17/Dez/2002

Versão final reapresentada em 21/Mai/2003

Aprovado em 25/Dez/2003 